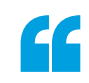

\section{Our soft matter computer} exploits soft materials and conducting fluids to deliver both analogue and digital computation

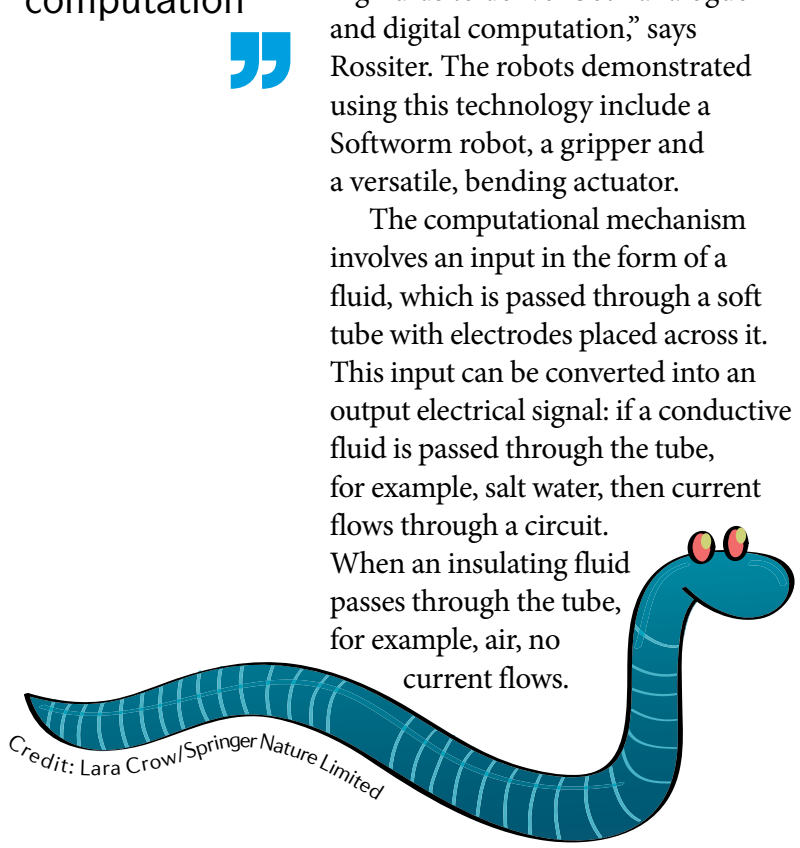

Soft robots are useful for automating delicate tasks such as sampling coral reefs or picking fruit. However, these robots are typically controlled by a hard silicon computer. Now, writing in Science Robotics, Jonathan Rossiter and colleagues report the control of soft robots using from soft materials.

"Our soft matter computer exploits soft materials and conducting fluids to deliver both analogue and digital computation," says Rossiter. The robots demonstrated using this technology include a Softworm robot, a gripper and ersatile, bending actuator. The computational mechanism involves an input in the form of a hh a soft output electrical signal: if a conductive luid is passed through the tube, flows through a circuit. When an insulating fluid passes through the tube, for example, air, no computational mechanisms made

\title{
SOFT ROBOTICS
}

\section{Moving with fluid control}

"This current is then used to generate movement in the robot or passes to the next computational component, or logic gate, in the computer," explains Rossiter.

The soft tubes of the computer are termed conductive fluid receptors (CFRs) by the researchers. A CFR can be triggered by a controller pulsing the fluids through the tube in a defined pattern, or it can be connected to another CFR that provides the input. By combining two CFRs either in series or parallel, different logic gates can be constructed.

The team integrate these soft computers into a Softworm robot, which crawls, inches or wriggles on a surface. To achieve these movements, shape memory alloy actuators are embedded into the body of the robot, and by switching on each actuator in turn, the robot moves its front and back feet, imitating a crawling pattern that moves the robot. These actuators are activated using two CFRs, which are triggered by a specific pattern of conducting and insulating fluids. This pattern of fluids is held in a fluidic input tape, which passes through the CFRs. Although the signal processing in the system is achieved using entirely soft components, a hard pump is still required to move the tape through the CFRs.
A further challenge in building an entirely soft computational system using this design is the need for an AC source to power the electrical circuit, made from hard components. An AC signal is needed because the salt water electrolyses and hence has lower conductivity when a DC signal is passed through. Rossiter suggests replacing the salt water with liquid metals, "as they are conductive in both DC and AC and do not suffer from any electrolysis effects."

For future systems, the researchers envisage more sophisticated soft computers that drive fully autonomous, untethered and intelligent soft robots. As soft robots are more adaptable than hard robots, owing to the flexibility of the material, their operation does not require large computational power. Although the soft matter computer may not be able to compete with the power of a traditional silicon computer, they are able to control soft robots in a useful way. Rossiter hopes to "extend soft matter computers to other applications, from reactive intelligent clothing to smart packaging." More generally, the combination of soft robots and computers that can control them may bring the field of soft robotics and artificial intelligence closer together.

Ankita Anirban

ORIGINAL ARTICLE Garrad, M. et al. A soft matter computer for robots. Sci. Robot. 4, eaaw6060 (2019) 